\title{
On entropy production in quantum statistical mechanics
}

\author{
V. Jakšićc ${ }^{1 *}$ and C.-A. Pillet ${ }^{2,3}$ \\ ${ }^{1}$ Department of Mathematics \\ Johns Hopkins University \\ 3400 N. Charles Street, 404 Krieger Hall \\ Baltimore, MD 21218, USA \\ ${ }^{2}$ PHYMAT \\ Université de Toulon, B.P. 132 \\ F-83957 La Garde Cedex, France \\ ${ }^{3}$ CPT-CNRS Luminy, Case 907 \\ F-13288 Marseille Cedex 9, France
}

August 3, 2000

\begin{abstract}
We propose a definition of entropy production in the framework of algebraic quantum statistical mechanics. We relate our definition to heat flows through the system. We also prove that entropy production is non-negative in natural nonequilibrium steady states.
\end{abstract}

${ }^{*}$ On leave from Department of Mathematics and Statistics, University of Ottawa, 585 King Edward Avenue, Ottawa, ON, K1N 6N5, Canada 


\section{Introduction}

Let $(\mathcal{O}, \tau)$ be a $C^{*}$-dynamical system, where $\mathcal{O}$ is a $C^{*}$-algebra with identity and $\tau$ a strongly continuous group of automorphisms of $\mathcal{O}$ (strong continuity means that the map $\mathbf{R} \ni t \mapsto \tau^{t}(A)$ is continuous in norm for each $\left.A \in \mathcal{O}\right)$. The elements of $\mathcal{O}$ describe observables of the physical system under consideration. The group $\tau$ specifies their time evolution. A physical state of the system is described by a mathematical state on $\mathcal{O}$, that is, a positive linear functional $\omega$ such that $\omega(\mathbf{1})=1$. The set $E(\mathcal{O})$ of all states on $\mathcal{O}$ is a convex, weak-* compact subset of the dual $\mathcal{O}^{*}$. A state $\omega$ is invariant under the group $\tau$ if $\omega \circ \tau^{t}=\omega$ for all $t$. For our purposes we assume that in addition to $(\mathcal{O}, \tau)$ we are given a $\tau$-invariant state $\omega$.

The triple $(\mathcal{O}, \tau, \omega)$ describes a physical system in a steady state. We are interested in effects of local perturbations on such system. A local perturbation is specified by a selfadjoint element $V$ of $\mathcal{O}$ and in what follows we fix such a $V$. The perturbed time evolution is given by

$$
\tau_{V}^{t}(A) \equiv \tau^{t}(A)+\sum_{n \geq 1} \mathrm{i}^{n} \int_{0}^{t} \mathrm{~d} t_{1} \int_{0}^{t_{1}} \mathrm{~d} t_{2} \ldots \int_{0}^{t_{n-1}} \mathrm{~d} t_{n}\left[\tau^{t_{n}}(V),\left[\ldots\left[\tau^{t_{1}}(V), A\right]\right]\right] .
$$

The pair $\left(\mathcal{O}, \tau_{V}\right)$ is also a $C^{*}$-dynamical system. Following Ruelle [Ru2], we call the weak- $*$ limit points of the set

$$
\left\{\frac{1}{T} \int_{0}^{T} \omega \circ \tau_{V}^{t} d t \mid T>0\right\} \subset E(\mathcal{O}),
$$

nonequilibrium steady states (NESS) of the locally perturbed system. The set $\Sigma_{V}^{+}(\omega)$ of $\operatorname{NESS}$ of $\left(\mathcal{O}, \tau_{V}\right)$ is a non-empty, compact subset of $E(\mathcal{O})$ whose elements are $\tau_{V}$-invariant.

Our first assumption is:

(A1) There exists a strongly continuous group $\sigma_{\omega}$ of automorphims of $\mathcal{O}$ such that $\omega$ is $\left(\sigma_{\omega},-1\right)$-KMS state.

Let $\delta_{\omega}$ be the generator of $\sigma_{\omega}$ (i.e. $\sigma_{\omega}^{t}=\mathrm{e}^{t \delta_{\omega}}$ ). We denote by $\mathcal{D}\left(\delta_{\omega}\right)$ the domain of $\delta_{\omega}$. $\mathcal{D}\left(\delta_{\omega}\right)$ is a norm-dense $*$-subalgebra of $\mathcal{O}$ and for $A, B \in \mathcal{D}\left(\delta_{\omega}\right)$,

$$
\delta_{\omega}(A)^{*}=\delta_{\omega}\left(A^{*}\right), \quad \delta_{\omega}(A B)=\delta_{\omega}(A) B+A \delta_{\omega}(B) .
$$

Our second assumption is:

(A2) $V \in \mathcal{D}\left(\delta_{\omega}\right)$.

We define the observable

$$
\sigma_{V} \equiv \delta_{\omega}(V),
$$

and for reasons which will soon become clear, we call

$$
\operatorname{Ep}_{V}(\eta) \equiv \eta\left(\sigma_{V}\right)
$$


the entropy production (with respect to the reference state $\omega$ ) of the perturbed system $\left(\mathcal{O}, \tau_{V}\right)$ in the state $\eta \in E(\mathcal{O})$. Note that $\sigma_{V}$, and hence $\operatorname{Ep}_{V}(\cdot)$, depend in a non-trivial way on the state $\omega$.

Let $\left(\mathcal{H}_{\omega}, \pi_{\omega}, \Omega_{\omega}\right)$ be the GNS representation of the algebra $\mathcal{O}$ associated to $\omega$, and let $\mathcal{N}_{\omega}$ be the set of $\pi_{\omega}$-normal states on $\mathcal{O}$, that is, the states represented by density matrices on $\mathcal{H}_{\omega}$. Any $\eta \in \mathcal{N}_{\omega}$ has a continuous extension to $\mathfrak{M} \equiv \pi_{\omega}(\mathcal{O})^{\prime \prime}$ which we denote by the same letter. For $\eta, \xi \in \mathcal{N}_{\omega}$, we denote by $\operatorname{Ent}(\eta \mid \xi)$ the relative entropy of $\eta$ with respect to $\xi$. (We use the definition of relative entropy given in [BR2], Definition 6.2.29. This definition differs by a sign and the order of factors from the original Araki's definition [Ar].)

Our main result, which justifies the above definition of entropy production, is:

Theorem 1.1 Assume that (A1) and (A2) hold. Then, for any faithful state $\eta \in \mathcal{N}_{\omega}$ such that $\operatorname{Ent}(\eta \mid \omega)>-\infty$, one has

$$
\operatorname{Ent}\left(\eta \circ \tau_{V}^{t} \mid \omega\right)=\operatorname{Ent}(\eta \mid \omega)-\int_{0}^{t} \operatorname{Ep}_{V}\left(\eta \circ \tau_{V}^{s}\right) d s .
$$

Remark. The same result (with the same proof) holds for $W^{*}$-dynamical systems.

In the rest of this section we will discuss some elementary properties of $\operatorname{Ep}_{V}(\cdot)$. Let $\omega_{V}^{+} \in \Sigma_{V}^{+}(\omega)$ and $T_{n} \rightarrow \infty$ be such that

$$
\lim _{n} \frac{1}{T_{n}} \int_{0}^{T_{n}} \omega \circ \tau_{V}^{t} d t=\omega_{V}^{+} .
$$

Then, with the particular choice $\eta=\omega$, Theorem 1.1 gives

$$
\lim _{n} \frac{1}{T_{n}} \operatorname{Ent}\left(\omega \circ \tau_{V}^{T_{n}} \mid \omega\right)=-\lim _{n} \frac{1}{T_{n}} \int_{0}^{T_{n}} \omega\left(\tau_{V}^{s}\left(\sigma_{V}\right)\right) d s=-\operatorname{Ep}_{V}\left(\omega_{V}^{+}\right)
$$

Since the relative entropy is non-positive, we immediately get

Theorem 1.2 Assume that (A1) and (A2) hold. Then, for any NESS $\omega_{V}^{+} \in \Sigma_{V}^{+}(\omega)$, one has

$$
\operatorname{Ep}_{V}\left(\omega_{V}^{+}\right) \geq 0
$$

With regard to (1.2), on physical grounds one expects that the ratio

$$
\operatorname{Ent}\left(\eta \circ \tau_{V}^{t} \mid \omega\right) / t
$$

becomes independent of the choice of the reference state $\omega$ as $t \rightarrow \infty$. More precisely, the following result holds: 
Proposition 1.3 Assume (A1) and that $\eta \in \mathcal{N}_{\omega}$ is faithful. Then there is a norm-dense set $\mathcal{N}_{\omega}^{\prime} \subset \mathcal{N}_{\omega}$ such that for $\omega^{\prime} \in \mathcal{N}_{\omega}^{\prime}$,

$$
\operatorname{Ent}\left(\eta \circ \tau_{V}^{t} \mid \omega^{\prime}\right)=\operatorname{Ent}\left(\eta \circ \tau_{V}^{t} \mid \omega\right)+O(1),
$$

as $t \rightarrow \infty$.

One also expects that in thermal equilibrium the entropy production is zero, that is, if $\eta \in \mathcal{N}_{\omega}$ is a $\left(\tau_{V}, \beta\right)$-KMS state then $\operatorname{Ep}_{V}(\eta)=0$. In fact, a much stronger result holds.

Proposition 1.4 Assume (A1), (A2) and that $\eta \in \mathcal{N}_{\omega}$ is a faithful, $\tau_{V}$-invariant state. Then

$$
\operatorname{Ep}_{V}(\eta)=0
$$

Remark. Again, this result also holds for $W^{*}$-dynamical systems.

Let $\mathcal{O}$ be the CAR algebra over $l^{2}\left(\mathbf{Z}^{3}\right)$ describing a free Fermi gas on the lattice $\mathbf{Z}^{3}$. Using some technical results proven in $[\mathrm{BM}]$ it is easy to construct a large class of quasifree states $\omega$ and local perturbations $V$ such that (A1)-(A2) hold, and that $\Sigma_{V}^{+}(\omega)$ consists of a single state $\omega_{V}^{+}$. In these examples, $\operatorname{Ep}_{V}\left(\omega_{V}^{+}\right)$can be computed perturbatively (similar calculations are done in [HTP]), and one easily constructs examples where $\operatorname{Ep}_{V}\left(\omega_{V}^{+}\right)>0$.

In the next example we relate entropy production to heat flows.

Consider two independent systems $\left(\mathcal{O}_{i}, \tau_{i}, \omega_{i}\right), i=1,2$, each of which is in thermal equilibrium at temperature $T_{i}$. This means that $\omega_{i}$ is a $\left(\tau_{i}, \beta_{i}\right)$-KMS state on $\mathcal{O}_{i}$ where $\beta_{i}=1 / T_{i}$. Let

$$
\mathcal{O}=\mathcal{O}_{1} \otimes \mathcal{O}_{2}, \quad \tau=\tau_{1} \otimes \tau_{2}, \quad \omega=\omega_{1} \otimes \omega_{2} .
$$

( $\otimes$ is the $C^{*}$-tensor product, see Section 2.7.2 in [BR1]). Let $\delta$ be the generator of $\tau$ and $\delta_{i}$ the generator of $\tau_{i}$. Obviously, $\delta=\delta_{1}+\delta_{2}$ (here we write $\delta_{1}$ for $\delta_{1} \otimes \mathbf{1}$, etc). Let $V \in \mathcal{O}$ be such that $V \in \mathcal{D}\left(\delta_{i}\right)$. Then

$$
\omega \circ \tau_{V}^{t}(V)-\omega(V)=\int_{0}^{t} \omega \circ \tau_{V}^{s}(\Phi) d s
$$

where $\Phi$ is defined by

$$
\tau_{V}^{t}(\Phi)=\frac{d}{d t} \tau_{V}^{t}(V)
$$

Obviously, $\Phi=\Phi_{1}+\Phi_{2}$ where $\Phi_{i} \equiv \delta_{i}(V)$ describes the energy flux out of the $i$-th system. Since the states $\omega_{i}$ are KMS, (A1) holds with $\delta_{\omega}=\delta_{\omega_{1}}+\delta_{\omega_{2}}$ and $\delta_{\omega_{i}}=-\beta_{i} \delta_{i}$. Therefore, (A1) and (A2) hold and

$$
\beta_{1} \Phi_{1}+\beta_{2} \Phi_{2}=-\sigma_{V}
$$

It follows that in a NESS $\omega_{V}^{+} \in \Sigma_{V}^{+}(\omega)$, the energy fluxes satisfy

$$
\frac{\omega_{V}^{+}\left(\Phi_{1}\right)}{T_{1}}+\frac{\omega_{V}^{+}\left(\Phi_{2}\right)}{T_{2}}=-\operatorname{Ep}_{V}\left(\omega_{V}^{+}\right) \leq 0
$$


Since $\omega_{V}^{+}\left(\Phi_{1}\right)+\omega_{V}^{+}\left(\Phi_{2}\right)=0$, if $T_{1}>T_{2}$, then $\Phi_{1} \geq 0$ and the heat flows from the hot to the cold reservoir. This calculation is easily generalized to the case where $N$-level quantum system is coupled to several independent thermal resevoirs.

We finish this section with the following remarks.

In [JP1] we prove an analog of Theorem 1.1 for time-dependent perturbations and discuss the relation between entropy production and the second law of thermodynamics.

In the forthcoming paper [JP2], we will study NESS, entropy production and heat flows for a model of an $N$-level quantum system coupled to several independent free Fermi gas reservoirs (similar models have been studied in [D, Ru1]).

The entropy production for quantum spin systems has been studied in the recent preprint [Ru2].

Acknowledgments. The research of the first author was partly supported by NSERC. Part of this work has been performed during a visit of the second author to University of Ottawa which was also supported by NSERC.

\section{Proofs}

We assume that the reader is familiar with the basic results of Tomita-Takesaki modular theory as discussed, for example, in [BR1, BR2, H, OP]. We begin by setting the notation and recalling some well-known facts.

$\left(\mathcal{H}_{\omega}, \pi_{\omega}, \Omega_{\omega}\right)$ denotes the GNS representation of the algebra $\mathcal{O}$ associated to $\omega$. By (A1), the vector $\Omega_{\omega}$ is cyclic and separating for $\mathfrak{M} \equiv \pi_{\omega}(\mathcal{O})^{\prime \prime}$. Moreover, (A1) implies that $\pi_{\omega}$ is injective. We respectively denote by $\Delta_{\omega}, J$ and $\mathcal{P}$ the modular operator, the modular conjugation and the natural cone canonically associated to the pair $\left(\mathfrak{M}, \Omega_{\omega}\right)$. We also adopt the shorthands $\mathcal{L}_{\omega}=\log \Delta_{\omega}$ and $j(A)=J A J$. Note that

$$
\pi_{\omega}\left(\sigma_{\omega}^{t}(A)\right)=\mathrm{e}^{\mathrm{i} t \mathcal{L}_{\omega}} \pi_{\omega}(A) \mathrm{e}^{-\mathrm{i} t \mathcal{L}_{\omega}} .
$$

With a slight abuse of notation, we write $\sigma_{\omega}^{t}(A)=\mathrm{e}^{\mathrm{i} t \mathcal{L}_{\omega}} A \mathrm{e}^{-\mathrm{i} t \mathcal{L}_{\omega}}$ for $A \in \mathfrak{M}$. By the Tomita-Takesaki theorem, $\sigma_{\omega}^{t}(\mathfrak{M})=\mathfrak{M}, j(\mathfrak{M})=\mathfrak{M}^{\prime}$ and

$$
\mathrm{e}^{\mathrm{i} t \mathcal{L}_{\omega}} J=J \mathrm{e}^{\mathrm{i} t \mathcal{L}_{\omega}} .
$$

The Liouvillean $L$ of the system $(\mathcal{O}, \tau, \omega)$ is the unique self-adjoint operator on $\mathcal{H}_{\omega}$ such that

$$
\begin{aligned}
\pi_{\omega}\left(\tau^{t}(A)\right) & =\mathrm{e}^{\mathrm{i} t L} \pi_{\omega}(A) \mathrm{e}^{-\mathrm{i} t L} \\
L \Omega_{\omega} & =0
\end{aligned}
$$

and one easily shows that

$$
\begin{aligned}
\mathrm{e}^{\mathrm{i} t L} \mathcal{L}_{\omega} & =\mathcal{L}_{\omega} \mathrm{e}^{\mathrm{i} t L} \\
\mathrm{e}^{\mathrm{i} t L} J & =J \mathrm{e}^{\mathrm{i} t L} .
\end{aligned}
$$


The self-adjoint operator

$$
L_{V} \equiv L+\pi_{\omega}(V)-j\left(\pi_{\omega}(V)\right),
$$

is uniquely specified by the following two requirements:

$$
\begin{aligned}
\pi_{\omega}\left(\tau_{V}^{t}(A)\right) & =\mathrm{e}^{\mathrm{i} t L_{V}} \pi_{\omega}(A) \mathrm{e}^{-\mathrm{i} t L_{V}} \\
\mathrm{e}^{-\mathrm{i} t L_{V}} \mathcal{P} & \subset \mathcal{P} .
\end{aligned}
$$

The dynamical groups $\tau$ and $\tau_{V}$ have natural extensions to $\mathfrak{M}$ for which we use the same notation. Note also that $J L_{V}+L_{V} J=0$, and therefore

$$
\mathrm{e}^{\mathrm{i} t L_{V}} J=J \mathrm{e}^{\mathrm{i} t L_{V}} .
$$

A state $\eta \in \mathcal{N}_{\omega}$ has a unique vector representative $\Omega_{\eta} \in \mathcal{P}$. Relations (2.6) yield that

$$
\Omega_{\eta \circ \tau_{V}^{t}}=\mathrm{e}^{-\mathrm{i} t L_{V}} \Omega_{\eta}
$$

The relative entropy of two faithful states $\eta, \xi \in \mathcal{N}_{\omega}$ is defined as

$$
\operatorname{Ent}(\eta \mid \xi) \equiv\left(\Omega_{\eta} \mid \log \Delta_{\xi \mid \eta} \Omega_{\eta}\right)
$$

where $\Delta_{\xi \mid \eta}$ is the relative modular operator. Relative entropy is more conveniently expressed in terms of the Radon-Nikodym cocycle $[D \xi: D \eta]^{s}$ as

$$
\operatorname{Ent}(\eta \mid \xi)=\lim _{s \downarrow 0} \frac{\eta\left([D \xi: D \eta]^{s}-\mathbf{1}\right)}{\mathrm{i} s} .
$$

Proof of Theorem 1.1. Let us denote by

$$
U(t) \equiv \mathrm{e}^{-\mathrm{i} t L} \mathrm{e}^{\mathrm{i} t\left(L+\pi_{\omega}(V)\right)},
$$

the propagator in the interaction representation. $U(t)$ is the unique solution of

$$
\frac{1}{\mathrm{i}} \frac{\mathrm{d}}{\mathrm{d} t} U(t)=\pi_{\omega}\left(\tau^{-t}(V)\right) U(t),
$$

with initial data $U(0)=\mathbf{1}$. It has a norm convergent Dyson expansion

$$
U(t)=\mathbf{1}+\sum_{n=1}^{\infty} \mathrm{i}^{n} \int_{0}^{t} d t_{1} \int_{0}^{t_{1}} d t_{2} \cdots \int_{0}^{t_{n-1}} d t_{n} \pi_{\omega}\left(\tau^{-t_{1}}(V)\right) \cdots \pi_{\omega}\left(\tau^{-t_{n}}(V)\right),
$$

from which we conclude that $U(t) \in \pi_{\omega}(\mathcal{O})$. With a slight abuse of notation, we will write $\delta_{\omega}$ for $\pi_{\omega} \circ \delta_{\omega}$. A simple calculation shows that $U(t) \in \mathcal{D}\left(\delta_{\omega}\right)$ and

$$
U^{*}(t) \delta_{\omega}(U(t))=-\delta_{\omega}\left(U^{*}(t)\right) U(t)=\mathrm{i} \int_{0}^{t} \pi_{\omega}\left(\tau_{V}^{-s}\left(\sigma_{V}\right)\right) d s
$$


We claim that

$$
\mathrm{e}^{-\mathrm{i} t L} \mathrm{e}^{\mathrm{i} t L_{V}}=U(t) j(U(t)) .
$$

To prove this fact, note that after differentiation with respect to $t$ both sides satisfy the same differential equation with the same initial condition at $t=0$.

To compute the relative entropy $\operatorname{Ent}\left(\eta \circ \tau_{V}^{t} \mid \omega\right)$, we will use Equation (2.9) and the fact that the Radon-Nikodym cocycle can be expressed as

$$
\left[D \omega: D \eta \circ \tau_{V}^{t}\right]^{s}=\Delta_{\omega \mid \omega \circ \tau_{V}^{t}}^{\mathrm{i} s} \Delta_{\eta \circ \tau_{V}^{t} \mid \omega \circ \tau_{V}^{t}}^{-\mathrm{i} s} .
$$

By definition of the relative modular operator, for any $A \in \mathfrak{M}$ we have

$$
J \Delta_{\eta \circ \tau_{V}^{t} \mid \omega \circ \tau_{V}^{t}}^{1 / 2} A \Omega_{\omega \circ \tau_{V}^{t}}=A^{*} \Omega_{\eta \circ \tau_{V}^{t}} .
$$

Using Relations (2.6) and (2.8), we further obtain

$$
J \Delta_{\eta \circ \tau_{V}^{t} \mid \omega \circ \tau_{V}^{t}}^{1 / 2} A \mathrm{e}^{-\mathrm{i} t L_{V}} \Omega_{\omega}=A^{*} \mathrm{e}^{-\mathrm{i} t L_{V}} \Omega_{\eta} .
$$

It follows that

$$
\begin{aligned}
J \Delta_{\eta \circ \tau_{V}^{t} \mid \omega \circ \tau_{V}^{t}}^{1 / 2} \mathrm{e}^{-\mathrm{i} t L_{V}} \tau_{V}^{t}(A) \Omega_{\omega} & =\mathrm{e}^{-\mathrm{i} t L_{V}} \tau_{V}^{t}(A)^{*} \Omega_{\eta} \\
& =\mathrm{e}^{-\mathrm{i} t L_{V}} J \Delta_{\eta \mid \omega}^{1 / 2} \tau_{V}^{t}(A) \Omega_{\omega} \\
& =J \mathrm{e}^{-\mathrm{i} t L_{V}} \Delta_{\eta \mid \omega}^{1 / 2} \tau_{V}^{t}(A) \Omega_{\omega},
\end{aligned}
$$

where we used (2.7). Since $\mathfrak{M} \Omega_{\omega}$ is a core for $\Delta_{\eta \mid \omega}^{1 / 2}$ and $\mathrm{e}^{-\mathrm{i} t L_{V}} \mathfrak{M} \Omega_{\omega}=\mathfrak{M} \Omega_{\omega \circ \tau_{V}^{t}}$ is a core for $\Delta_{\eta \circ \tau_{V}^{t} \mid \omega \circ \tau_{V}^{t}}^{1 / 2}$, we derive the relation

$$
\Delta_{\eta \circ \tau_{V}^{t} \mid \omega \circ \tau_{V}^{t}}=\mathrm{e}^{-\mathrm{i} t L_{V}} \Delta_{\eta \mid \omega} \mathrm{e}^{\mathrm{i} t L_{V}}
$$

We now deal with $\Delta_{\omega \mid \omega \circ \tau_{V}^{t}}$. First, for any $A \in \mathfrak{M}$,

$$
J \Delta_{\omega \mid \omega \circ \tau_{V}^{t}}^{1 / 2} A \Omega_{\omega \circ \tau_{V}^{t}}=A^{*} \Omega_{\omega}
$$

Equations (2.8), (2.11) and (2.4) yield that

$$
\Omega_{\omega \circ \tau_{V}^{t}}=U^{*}(t) j\left(U^{*}(t)\right) \Omega_{\omega},
$$

and since $j\left(U^{*}(t)\right) \in \mathfrak{M}^{\prime}$, we derive

$$
\begin{aligned}
J \Delta_{\omega \mid \omega \circ \tau_{V}^{t}}^{1 / 2} j\left(U^{*}(t)\right) A U^{*}(t) \Omega_{\omega} & =A^{*} \Omega_{\omega} \\
& =U^{*}(t)\left(A U^{*}(t)\right)^{*} \Omega_{\omega} \\
& =U^{*}(t) J \Delta_{\omega}^{1 / 2} A U^{*}(t) \Omega_{\omega} \\
& =J j\left(U^{*}(t)\right) \Delta_{\omega}^{1 / 2} A U^{*}(t) \Omega_{\omega} .
\end{aligned}
$$


Since $\mathfrak{M} \Omega_{\omega}$ is a core of $\Delta_{\omega}^{1 / 2}$ and $j\left(U^{*}(t)\right) \mathfrak{M} \Omega_{\omega}=\mathfrak{M} \Omega_{\omega \circ \tau_{V}^{t}}$ is a core of $\Delta_{\omega \mid \omega \circ \tau_{V}^{t}}^{1 / 2}$ we conclude that

$$
\Delta_{\omega \mid \omega \circ \tau_{V}^{t}}=j\left(U^{*}(t)\right) \Delta_{\omega} j(U(t)) .
$$

Going back to (2.12), we derive from Equations (2.13) and (2.14)

$$
\begin{aligned}
{\left[D \omega: D \eta \circ \tau_{V}^{t}\right]^{s} } & =j\left(U^{*}(t)\right) \Delta_{\omega}^{\mathrm{i} s} j(U(t)) \mathrm{e}^{-\mathrm{i} t L_{V}} \Delta_{\eta \mid \omega}^{-\mathrm{i} s} \mathrm{e}^{\mathrm{i} t L_{V}} \\
& =j\left(U^{*}(t)\right) \Delta_{\omega}^{\mathrm{i} s} U^{*}(t) \mathrm{e}^{-\mathrm{i} t L} \Delta_{\eta \mid \omega}^{-\mathrm{i} s} \mathrm{e}^{\mathrm{i} t L_{V}} \\
& =j\left(U^{*}(t)\right) \sigma_{\omega}^{s}\left(U^{*}(t)\right) \mathrm{e}^{-\mathrm{i} t L} \Delta_{\omega}^{\mathrm{i} s} \Delta_{\eta \mid \omega}^{-\mathrm{i} s} \mathrm{e}^{\mathrm{i} t L_{V}},
\end{aligned}
$$

where we used (2.11) and (2.5). Since $\sigma_{\omega}^{s}\left(U^{*}(t)\right) \in \mathfrak{M}$, it commutes with $j\left(U^{*}(t)\right)$ and another application of (2.11) (together with Relation (2.12) at $t=0$ ) gives

$$
\left[D \omega: D \eta \circ \tau_{V}^{t}\right]^{s}=\sigma_{\omega}^{s}\left(U^{*}(t)\right) U(t) \tau_{V}^{-t}\left([D \omega: D \eta]^{s}\right) .
$$

We can therefore write

$$
\eta \circ \tau_{V}^{t}\left(\left[D \omega: D \eta \circ \tau_{V}^{t}\right]^{s}\right)=\left(\mathrm{e}^{\mathrm{i} t L_{V}} U^{*}(t) \sigma_{\omega}^{s}(U(t)) \mathrm{e}^{-\mathrm{i} t L_{V}} \Omega_{\eta},[D \omega: D \eta]^{s} \Omega_{\eta}\right) .
$$

Since $U(t) \in \mathcal{D}\left(\delta_{\omega}\right)$, the estimate

$$
\mathrm{e}^{\mathrm{i} t L_{V}} U^{*}(t) \sigma_{\omega}^{s}(U(t)) \mathrm{e}^{-\mathrm{i} t L_{V}} \Omega_{\eta}=\Omega_{\eta}+s \mathrm{e}^{\mathrm{i} t L_{V}} U^{*}(t) \delta_{\omega}(U(t)) \mathrm{e}^{-\mathrm{i} t L_{V}} \Omega_{\eta}+\mathrm{o}(s),
$$

holds in the norm of $\mathcal{H}_{\omega}$ as $s \downarrow 0$. Furthermore, Equation (2.10) is easily rewritten as

$$
\mathrm{e}^{\mathrm{i} t L_{V}} U^{*}(t) \delta_{\omega}(U(t)) \mathrm{e}^{-\mathrm{i} t L_{V}}=\mathrm{i} \int_{0}^{t} \pi_{\omega}\left(\tau_{V}^{u}\left(\sigma_{V}\right)\right) d u .
$$

Equation (2.16) leads to the estimate

$$
\begin{aligned}
\eta \circ \tau_{V}^{t}\left(\left[D \omega: D \eta \circ \tau_{V}^{t}\right]^{s}\right) & =\eta\left([D \omega: D \eta]^{s}\right) \\
& -\mathrm{i} s \int_{0}^{t}\left(\Omega_{\eta}, \pi_{\omega}\left(\tau_{V}^{u}\left(\sigma_{V}\right)\right)[D \omega: D \eta]^{s} \Omega_{\eta}\right) d u+\mathrm{o}(s),
\end{aligned}
$$

as $s \downarrow 0$. Since the cocyle $[D \omega: D \eta]^{s}$ is strongly continuous, insertion in Equation (2.9) gives the result.

Proof of Proposition 1.3. For any self-adjoint $P \in \mathfrak{M}$ we define a group of automorphisms of $\mathfrak{M}$ by

$$
\sigma_{P}^{t}(A)=\mathrm{e}^{\mathrm{i} t\left(\mathcal{L}_{\omega}+P\right)} A \mathrm{e}^{-\mathrm{i} t\left(\mathcal{L}_{\omega}+P\right)} .
$$

Araki's perturbation theory yields that there is a state $\omega_{P} \in \mathcal{N}_{\omega}$ which is a $\left(\sigma_{P},-1\right)$-KMS state. Let $\mathcal{N}_{\omega}^{\prime}$ be the set of all states obtained in this manner. It is well-known that $\mathcal{N}_{\omega}$ is dense in $\mathcal{N}_{\omega}$ (see, e.g., [R]). By the result of Araki (see Proposition 6.2.32 in [BR2]),

$$
\operatorname{Ent}\left(\eta \circ \tau_{V}^{t} \mid \omega_{P}\right)=\operatorname{Ent}\left(\eta \circ \tau_{V}^{t} \mid \omega\right)+\eta\left(\tau_{V}^{t}(P)\right)-\log \left\|\mathrm{e}^{\left(\mathcal{L}_{\omega}+P\right) / 2} \Omega_{\omega}\right\|^{2} .
$$


The statement follows from this relation, the obvious estimate $\left|\eta\left(\tau_{V}^{t}(P)\right)\right| \leq\|P\|$ and the fact that $0<\left\|\mathrm{e}^{\left(\mathcal{L}_{\omega}+P\right) / 2} \Omega_{\omega}\right\|<\infty$.

Proof of Proposition 1.4. Since $\eta \circ \tau_{V}^{t}=\eta$, Relation (2.17) yields that for all $s>0$,

$$
\int_{0}^{t}\left(\Omega_{\eta}, \pi_{\omega}\left(\tau_{V}^{u}\left(\sigma_{V}\right)\right)[D \omega: D \eta]^{s} \Omega_{\eta}\right) d u=o(1)
$$

Taking $s \downarrow 0$ we get that for all $t$,

$$
\int_{0}^{t} \eta\left(\sigma_{V}\right) d u=t \eta\left(\sigma_{V}\right)=0
$$

and so $\operatorname{Ep}_{V}(\eta)=0$.

\section{References}

[Ar] Araki, H.: Relative entropy of states of von Neumann algebras, Pub. R.I.M.S., Kyoto Univ. 11, 809 (1976).

[BM] Botvich, D.D., Malyshev, V.A.: Unitary equivalence of temperature dynamics for ideal and locally perturbed Fermi-gas, Commun. Math. Phys. 91, 301 (1983).

[BR1] Brattelli, O, Robinson, D. W.: Operator Algebras and Quantum Statistical Mechanics 1. Springer-Verlag, Berlin, second edition (1987)

[BR2] Brattelli, O, Robinson, D. W.: Operator Algebras and Quantum Statistical Mechanics 2. Springer-Verlag, Berlin, second edition (1996).

[D] Davies, E.B.: Markovian master equations, Commun. Math. Phys. 39, 91 (1974).

[H] Haag, R.: Local Quantum Physics. Springer-Verlag, Berlin (1993).

[HTP] Haag, R., Trych-Pohlmeyer E.: Stability properties of equilibrium states, Commun. Math. Phys. 56, 213 (1977).

[JP1] Jakšić, V., Pillet, C.-A.: On entropy production in quantum statistical mechanics II. Time-dependent perturbations, preprint.

[JP2] Jakšić, V., Pillet, C.-A.: In preparation.

[OP] Ohya, M., Petz, D.: Quantum Entropy and its Use, Springer-Verlag, Berlin (1993).

[R] Robinson, D.W.: $C^{*}$-algebras in quantum statistical mechanics, in $C^{*}$-algebras and their Applications to Statistical Mechanics and Quantum Field Theory, (D. Kastler editor), Amsterdam, North-Holand (1976).

[Ru1] Ruelle, D.: Natural nonequilibrium states in quantum statistical mechanics, J. Stat. Phys. 98, 57 (2000).

[Ru2] Ruelle, D.: Entropy production in quantum spin systems, preprint. 